\title{
Indirect CLEM - From Structural Insights to Novel Therapy for Atrial Fibrillation
}

Louisa Mezache ${ }^{1}$, Heather Struckman ${ }^{2}$, Anna Phillipsa ${ }^{1}$, Stephen Baine ${ }^{1}$, Amara Greer-Short ${ }^{1}$, Sandor Gyorke $^{1}$, Przemyslaw Radwanski ${ }^{1}$, Thomas Hund ${ }^{1}$ and Rengasayee Veeraraghavan ${ }^{1}$

${ }^{1}$ The Ohio State University, Columbus, Ohio, United States, ${ }^{2}$ The Ohio State University, Hilliard, Ohio, United States

Atrial fibrillation (AF), the most common arrhythmia, is a condition of the heart in which the atria beat irregularly and rapidly, and poses a potential risk for stroke and heart failure. AF affects approximately $3 \%$ of the US population and contributes a significant cost burden to the healthcare system. The current available treatments entail managing symptoms, and while AF begets AF, the mechanism of progression is unclear.

$\mathrm{AF}$ is associated with inflammation and vascular dysfunction and AF patients have elevated levels of vascular endothelial growth factor (VEGF-A; 90-580 pg/ml), which promotes vascular leak and edema [1-3]. We have previously identified edema-induced disruption of sodium channel (Nav1.5) -rich intercalated disk (ID) nanodomains as a novel arrhythmia mechanism. We hypothesized that (i) elevated VEGF-A levels promote AF by disrupting ID nanodomains, and slowing atrial conduction, and (ii) protection of the vascular barrier can prevent these arrhythmias.

To identify the structural underpinnings of disease mechanism, we employed a new tool we developed called indirect correlative light and electron microscopy (iCLEM). With iCLEM, we were able to correlate the cardiac myocyte ultrastructure, assessed using transmission electron microscopy (TEM), with its molecular organization, assessed using sub-diffraction confocal imaging (sDCI) and stochastic optical reconstruction microscopy (STORM). Briefly, data from these diverse methods were correlated by overlaying distributions of measurements collected relative to structural fiducials identifiable using both light and electron microscopy. TEM images revealed ID nanodomain swelling, near both gap junctions (perinexi; $64 \pm 9 \mathrm{~nm}$ vs $17 \pm 1 \mathrm{~nm})$ and mechanical junctions $(63 \pm 4 \mathrm{~nm}$ vs $27 \pm 2 \mathrm{~nm})$ in VEGF-A-treated hearts

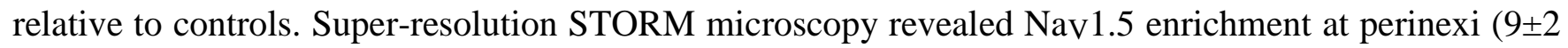
fold) and $\mathrm{N}$-cadherin-rich sites ( $7 \pm 1$ fold) relative to non-junctional ID sites in control hearts. VEGF-A reduced Nav1.5 enrichment at both sites ( $6 \pm 1$ and $4 \pm 1$ fold, respectively), consistent with Nav1.5 translocation from ID nanodomains. Atrial conduction, assessed by optical mapping, was slowed by VEGF-A $(10 \pm 0.4 \mathrm{~cm} / \mathrm{s}$ vs $21.3 \pm 1.3 \mathrm{~cm} / \mathrm{s}$ at baseline $)$. VEGF-A increased atrial arrhythmia burden both ex vivo ( $80 \%$ vs $0 \%$ in vehicle controls) and in vivo (70\% vs $20 \%$ in vehicle controls). Overall, median in vivo arrhythmia burden (seconds of atrial arrhythmia per hour of observation) was significantly higher in VEGF-A-treated mice $(7.49 \pm 10.98 \mathrm{~s} / \mathrm{hr})$ than in untreated controls $(0 \pm 7.07 \mathrm{~s} / \mathrm{hr})$. These results highlight VEGF-A-induced vascular leak as a novel atrial arrhythmia mechanism.

Next, we identified two molecular targets for vascular barrier protection, connexin43 (Cx43) and pannexin-1 (Panx1) [4,5]. In vascular endothelial cells, the opening of connexin43 hemichannels and pannexin channels, providing electrical and chemical coupling between cells and extracellular space, has been implicated in cytokine-induced vascular leak. Therefore, we assessed the antiarrhythmic potential of connexin43 hemichannels and pannexin channel inhibitors in VEGF-A-treated mice. Treatment with aCT11 (100 $\mu \mathrm{M}$; a Cx43 mimetic peptide) decreased both incidence (40\%) and duration $(1.45 \pm 7.65 \mathrm{~s})$ of VEGF-A-induced arrhythmias. Overall, $\alpha \mathrm{CT} 11$ reduced in vivo arrhythmia burden to $0 \pm 6.07 \mathrm{~s} / \mathrm{hr}$. Panx1- 
IL2 (a peptide inhibitor of Panx1 channels) treatment decreased also in vivo arrhythmia burden ( $0 \pm 15.57$ s/hr with 1.6 $\mu \mathrm{M}$ Panx1-IL2).

iCLEM allowed us to identify VEGF-A-induced vascular leak as a novel atrial arrhythmia mechanism and pinpoint the vascular barrier as a therapeutic target for AF prevention.

References

1. NA Chung et al., Stroke 33 (2002), p. 2187.

2. J Li et al., Heart rhythm: The Official Journal of the Heart Rhythm Society 7 (2010), p. 438.

3. Y Seko et al., Jpn Heart J 41 (2000), p. 27.

4. J Jiang et al., JAHA 8 (2019), e012385.

5. M Billaud et al., Sci Signal 8 (2015), p.364. 